\section{DISCRIM: A BASIC program to perform discriminant function analysis}

\section{STEPHEN POWERS \\ Tucson Unified School District, Tucson, Arizona}

Discriminant analysis is a procedure that utilizes a set of discriminating variables to maximally discriminate members of two or more groups. The resulting discriminant function enables the researcher to decide to which group each member probably belongs. The two main uses of discriminant analysis are to classify and diagnose and to study the relations among variables in different populations and samples (Kerlinger, 1964). This paper describes DISCRIM, a microcomputer program that performs a discriminant function analysis.

The analysis in the program follows the description by Tatsouka (1970, 1971). It utilizes two major subroutines: (1) the Gauss-Jordan method of matrix inversion, which was adapted from Cooley and Lohnes (1971), and (2) a procedure that computes eigenvalues of a nonsymmetric or symmetric matrix (Heilborn, 1981).

Input. Data are placed into the program with DATA statements prior to execution. The user specifies parameters of the analysis, such as the number of subjects in each group, the number of groups, and the number of variables. The user may select to output group means, standard deviations, within-groups sums of squares, and cross-products (SSCP) matrices, as well as pooled within-groups, between-groups, and total SSCP matrices.

Output. The program calculates and presents stan-

The author's mailing address is: Legal and Research Services, Tucson Unified School District, P.O. Box 40400, Tucson, AZ 85717. dardized and unstandardized discriminant weights for each discriminant function. In addition, for each function, associated eigenvalues, percentages of between-groups variance accounted for, canonical correlations, and Wilks's lambdas are printed. Barlett's tests of overall discrimination and residual discrimination are also presented.

Program Language and Requirements. This program is written in TRS-80 Level II disk BASIC. It has been implemented on a $64 \mathrm{~K}$ TRS-80 Model IV microcomputer utilizing a TRS-DOS version 6.00 operating environment. Approximately $18 \mathrm{~K}$ of memory is required to accommodate the program. TRS-80 BASIC is similar to other BASIC dialects, and therefore, with minor modification, the program can easily be adapted to other systems.

Limitations. The program may require doubleprecision numbers with large data sets, which may cause an arithmetic overflow to the internal representation of floating-point numbers on the TRS-80. The range of numbers is approximately $1.7 \times 10^{-38}$ to $1.7 \times 10^{+38}$. This modification can be easily implemented.

Availability. A progam listing is available without charge from the author.

\section{REFERENCES}

COOLEY, W. W., \& LOHNes, P. R. (1971). Multivariate data analysis. New York: Wiley.

HEILBORN, J. (1981). Science and engineering programs: Apple II edition. Berkeley, CA: Osborn/McGraw-Hill.

KerLinger, F. N. (1964). Foundations of behavioral research (2nd ed.). New York: Holt, Rinehart \& Winston.

TatsuoKa, M. M. (1970). Discriminant analysis: The study of group differences. Champaign, IL: Institute for Personality and Ability Testing.

Tatsouka, M. M. (1971). Multivariate analysis; Techniques for educational and psychological research. New York: Wiley.

(Revision accepted for publication November 14, 1984.) 